\title{
TOXICIDAD DEL CROMO EN SEDIMENTO USANDO Donax obesulus Reeve 1854 (PELECYPODA: DONACIDAE)
}

\section{CHROMIUM TOXICITY IN A SEDIMENT USING Donax obesulus Reeve 1854 (PELECYPODA: DONACIDAE)}

\author{
Julio Lacma ${ }^{1}$, José Iannacone $e^{1,2}$ y Giovanna Vera ${ }^{3}$
}

\section{Resumen}

La evaluación de la toxicidad del cromo en los sedimentos marinos en el Perú es un tema de interés emergente. Se evaluó la concentración letal media del cromo $\left(\mathrm{Cr}^{+6}\right)$ usando el bivalvo marino Donax obesulus Reeve 1854 (Pelecypoda: Donacidae) a las 24 h, 48 h y 96 h de exposición, también fue estudiada la capacidad de enterramiento de D. obesulus sobre el sedimento frente al cromo $\left(\mathrm{Cr}^{+6}\right)$. Para las condiciones de aclimatación y cría de $D$. obesulus se empleó a la microalga marina Dunaliella tertiolecta Butcher. El bioensayo se realizó con adultos de $D$. obesulus de $22.4 \mathrm{~mm} \pm 1.5 \mathrm{~mm}$ La concentración letal media $\left(\mathrm{CL}_{50}\right)$ del cromo $\mathrm{Cr}^{+6}$ en el sedimento marino usando a $D$. obesulus fue de $410.4 \mathrm{mg} \cdot \mathrm{kg}^{-1}, 156.1 \mathrm{mg} \cdot \mathrm{kg}^{-1}$ y $61.12 \mathrm{mg} \cdot \mathrm{kg}^{-1}$ a 24 h, 48 h y 96 h de exposición, respectivamente. El valor guía propuesto por Long et al. (1995) para el cromo en el sedimento acuático es de $370 \mathrm{mg} \cdot \mathrm{kg}^{-1}$. Los resultados obtenidos en términos de $\mathrm{CL}_{50}$ a $48 \mathrm{~h}$ y $96 \mathrm{~h}$ para $D$. obesulus, expuestos al cromo, fueron $57.8 \%$ y $83.5 \%$ menores que el valor guía, respectivamente. El cromo presenta un notable efecto subletal sobre la capacidad de enterramiento de $D$. obesulus a las $48 \mathrm{~h}$ de exposición. El protocolo usando D. obesulus tiene como ventaja, además de su sensibilidad, el fácil uso y la simplicidad en la lectura, su bajo costo y su realismo ecológico.

Palabras claves: bioensayo, ecotoxicología, pelecípodo, cromo, Donax obesulus, sedimento

\begin{abstract}
Chromium toxicity assessment of marine sediments in Peru is a topic of increasing interest. Mean lethal concentration of chromium $\left(\mathrm{Cr}^{+6}\right)$ using the marine bivalve Donax obesulus Reeve 1854 (Pelecypoda: Donacidae) at $24 \mathrm{~h}, 48 \mathrm{~h}$ and $96 \mathrm{~h}$ of exposure was assessed, as well as its burying ability in a chromium $\left(\mathrm{Cr}^{+6}\right)$ sediment. For $D$. obesulus acclimatization and rearing the marine microalga Dunaliella tertiolecta Butcher was employed. The assay was performed with $22.4 \mathrm{~mm} \pm 1.5 \mathrm{~mm} \mathrm{D}$. obesulus adults. Mean lethal concentration $\left(\mathrm{LC}_{50}\right)$ in chromium $\mathrm{Cr}^{+6} \mathrm{D}$. obesulus marine sediment was $410.4 \mathrm{mg} \cdot \mathrm{kg}^{-1}, 156.1 \mathrm{mg} \cdot \mathrm{kg}^{-1}$ and $61.12 \mathrm{mg} \cdot \mathrm{kg}^{-1}$ at $24 \mathrm{~h}, 48 \mathrm{~h}$ and $96 \mathrm{~h}$ of exposure, respectively. A guideline value proposed by Long et al. (1995) for chromium in an aquatic sediment is $370 \mathrm{mg} \cdot \mathrm{kg}^{-1}$. The results obtained in terms of $\mathrm{LC}_{50}$ at $48 \mathrm{~h}$ and $96 \mathrm{~h}$ for $D$. obesulus, exposed to chromium, were $57.8 \%$ and $83.5 \%$, lower than the guideline value, respectively. Chromium presented a notorious sublethal effect on $D$. obesulus burying ability at 48 $\mathrm{h}$ of exposure. The protocol using $D$. obesulus has advantages in terms of sensibility, easy use, simplicity of reading, low cost and ecological realism.
\end{abstract}

Key Words: bioassay, ecotoxicology, pelecypod, chromium, Donax obesulus, sediment

\section{Introducción}

Las industrias mineras ubicadas en zonas alejadas a la costa peruana eliminan sus descargas contaminantes a cursos secos que después de un largo recorrido llegan a las aguas marítimas, donde los residuos minerales de dichos procesos sedimentan, destruyendo la biota que se desarrolla este ecosistema (Malnati, 1982). La disposición de relaves de las minas en el mar causa un fuerte impacto negativo sobre la fisiografía de la línea costera, así como en la contaminación de organismos de la comunidad bentónica de importancia ecológica y económica (Cripe, 2006). La contaminación de los sedimentos tiene una connotación especial, debido a que las condiciones fisicoquímicas del agua, los tóxicos tienden a acumularse en ellos, constituyéndose éstos el mayor sumidero para los contaminantes persistentes, exponiéndolos a los organismos acuáticos y alterando las cadenas tróficas (Del Valls et al., 1998; Chai et al., 2006; Dornfeld et al., 2006; Portela et al., 2006). El metal pesado cromo ha sido catalogado en la "lista Gris “, conjuntamente con otros metales, y solo debería ser descargado al medio acuático con extremas medidas de mitigación y seguridad. El cromo se origina en procesos de la industria del papel y cartón, petroquímicos, compuestos orgánicos, soda cáustica, abonos, refinerías, metalúrgica, metales diferentes al hierro, industria de autos y aviones, vidrio, cemento, 
asbesto, textiles, cueros, plantas a vapor (calderas), pigmentos, pinturas, cerámicas, fungicidas, ladrillos a prueba de fuego y catálisis. Los componentes cromados también son utilizados para control en sistemas de calentamiento y enfriamiento (Alayo \& Iannacone, 2000; Vera et al., 2001; Alayo et al., 2004; Becker et al., 2006; Roling et al., 2006).

Los moluscos están ampliamente distribuidos en todo nuestro litoral y tienen un gran valor alimenticio y ecológico (Sánchez \& Vera, 2001) y constituyen eslabones en las cadenas alimenticias de los vertebrados (Álamo \& Valdivieso, 1997). Muchos moluscos son adecuados para evaluar la toxicidad en pruebas a corto y largo plazo (APHA, 1995). Así, los ensayos ecotoxicológicos de sedimentos con bivalvos proporcionan información relevante (Del Valls et al., 1998). La información sobre la toxicidad sirve de base para la evaluación del riesgo ecológico, esto es, la probabilidad de ocurrencia de efectos negativos en el ecosistema marino (Gaete et al., 1996; Ong \& Din, 2001; Iannacone \& Alvariño, 2003).

En el Perú, algunos pelecípodos donácidos son fuente de alimento para el hombre, figurando en las estadísticas pesqueras como "palabritas" o "concha blanca", siendo dominante la especie Donax obesulus Reeve 1854 (Estrella et al., 2000). Esta especie se distingue de otros donácidos que habitan en el país, por la presencia de puntuaciones en las estrías del umbo, las que se desvían hacia los extremos y tiene un lomo posterior obtuso y un ligamento corto (Paredes \& Cardozo, 2001). Además está descrita en listas sistemáticas de moluscos bivalvos de importancia comercial y ecológica (Paredes et al., 1999; Álamo \& Valdivieso, 1997). Coan (1983) establece que Donax peruvianus Deshayes 1855 es un complejo de especies que incluye D. obesulus y Donax marincovichi Coan 1983. Sin embargo, en nuestro país en las estadísticas pesqueras se sigue considerando a estas dos especies como si fuese una sola, utilizando el nombre de $D$. peruvianus. Se han realizado algunos estudios sobre la madurez sexual, reproducción y algunas consideraciones ecológicas de D. peruvianus (Huaraz \& Ishiyama, 1980; Talledo, 1980; Talledo et al., 1987) así como su rol ecológico (Tarazona et al., 1986). Donax obesulus tiene distribución en las siguientes localidades peruanas: Tumbes (El Benito, Hueso de Ballena, Puerto Pizarro, Zorritos, Bocapan, Peñas Redondas, Cancas), Piura (Mancora, Los Órganos, Cabo Blanco, Lobitos, Colan), Lambayeque (Cherrepe), Ancash (Las Salinas de Chimus), Lima (Santa María) y su hábitat esta localizado en el intermareal y submareal arenoso (Paredes \& Cardozo, 2001).

La presente investigación tuvo como principales objetivos: 1) determinar la concentración efectiva media del metal del cromo en sedimentos marinos usando al molusco $D$. obesulus a $24 \mathrm{~h}, 48 \mathrm{~h}$ y $96 \mathrm{~h}$ de exposición, y 2) evaluar alteraciones en el comportamiento de $D$. obesulus frente al cromo en sedimentos a 24 h y 48 h de exposición.

\section{Materiales y métodos}

\section{Colecta de organismo prueba}

Se procedió a la colección, identificación y cría estandarizada de $D$. obesulus. 350 ejemplares de $D$. obesulus fueron colectados a $20 \mathrm{~m}$ de distancia de la orilla y hasta un máximo de $\approx 1 \mathrm{~m}$ de profundidad en la zona intermareal de la playa Herbay Bajo $\left(13^{\circ} 09^{\prime}\right.$ $\mathrm{S} ; 76^{\circ} 22^{\prime} \mathrm{W}$ ), altura del km 152 de la Panamericana Sur, Provincia de Cañete, Departamento de Lima, Perú. La playa de Herbay Bajo es arenosa y semiprotegida. La colecta se realizó en forma artesanal empleando una malla de $5 \mathrm{~mm}$ de diámetro. $D$. obesulus se encontró enterrado en la arena a aproximadamente a $3 \mathrm{~cm}$ de profundidad. Los 350 ejemplares fueron transportados con el sedimento y el agua de mar del mismo lugar de colecta en una caja térmica hasta el laboratorio.

Los especímenes de D. obesulus conocidos vernacularmente en la zona como "morocho" o "concha mariposa" fueron identificados empleando las claves de Coan (1983) y de Álamo \& Valdivieso (1997).

\section{Aclimatación de organismos}

Los especímenes adultos de 17 a $25 \mathrm{~mm}$ de longitud valvar fueron colocados en bandejas de $36 \mathrm{~L}$ de capacidad, conteniendo cada una de 2 a $3 \mathrm{~cm}$ de sedimento marino del lugar de colecta, es decir 15.6 $\mathrm{kg}$ bandeja ${ }^{-1}$. Luego se le agregó $35 \mathrm{~L}$ de agua de mar UV filtrada, la cual fue aireada permanentemente mediante piedras difusoras y por una bomba de aire (Blower®) y estas bandejas fueron colocadas una mesa termorreguladora que les proveyó una temperatura adecuada de $19 \pm 2{ }^{\circ} \mathrm{C}$. La aclimatación de los organismos previa a los bioensayos en el laboratorio fue de aproximadamente un mes (APHA, 1995).

El oxígeno disuelto $\left(\mathrm{mg} \cdot \mathrm{L}^{-1}\right)$ se determinó usando el oxímetro Modelo HANNA HI 9143®, el pH se midió usando un Potenciómetro modelo OAKTON ${ }^{\circledR}$, la salinidad (ups) se determinó usando el refractómetro Modelo ATAGO S-28® y la temperatura $\left({ }^{\circ} \mathrm{C}\right)$ se midió mediante un termómetro modelo PRECISIÓN - DIGITAL ${ }^{\circledR}$. Todos los parámetros se midieron en las cuatro réplicas al inicio del ensayo y cada $24 \mathrm{~h}$.

Durante la aclimatación se encontró un $\mathrm{pH}$ de 7.90 $\pm 0.12, \mathrm{OD}=7.10 \pm 0.58 \mathrm{mg} \mathrm{L}^{-1}$ y una salinidad de $33.75 \pm 0.77$ ups. Estos parámetros fueron medidos diariamente antes de cada recambio de agua y de alimentación. D. obesulus se alimentó durante su acondicionamiento y aclimatación por separado solamente con la especie de microalga más eficiente, en recipientes de 10 a $20 \mathrm{~L}$. 


\section{Tasa de filtración}

Se realizaron cultivos de tres microalgas: Skeletonema costatum (Greville) Cleve, Dunaliella tertiolecta Butcher y Chaetoceros gracilis Schütt para determinar cual presentaba la mayor tasa de filtración en $D$. obesulus. Se usaron las cepas de las microalgas $S$. costatum, D. tertiolecta y Ch. gracilis cultivadas en medio f/2 de Guillard, luego transferidas a cultivos intermedios (matraces de $1 \mathrm{~L}$ ) y masivos (bombonas de $5 \mathrm{~L}$ y $20 \mathrm{~L}$ ), conservadas en cámaras especiales con aire acondicionado y con enfriadores, a una temperatura controlada de $23 \pm 2{ }^{\circ} \mathrm{C}$, con aireación constante, iluminación artificial continua de $24 \mathrm{~h}$ y salinidad de 40 ups. Luego, se determinó la tasa de filtración, para lo cual, se utilizó 15 especímenes adultos de los controles para las tres especies de microalgas con 5 réplicas, la prueba de filtración se realizó en vasos de $350 \mathrm{~mL}$, con $100 \mathrm{~g}$ de sedimento esterilizado, aireación constante, con $100 \mathrm{~mL}$ agua UV filtrada con poro de $0.45 \mu \mathrm{m}$ y $100 \mathrm{~mL}$ de cada especie de microalgas. La prueba tuvo una duración de $1 \mathrm{~h}$, con lecturas a las 0.30 y 60 min. Se utilizó una micropipeta para la toma de las muestras y preservación en viales de $3 \mathrm{~mL}$ con agua-alcohol (3:1) y añadiendo 5 gotas de lugol. Finalmente se procedió a la cuantificación usando un contómetro y una cámara de Neubauer ${ }^{\circledR}$.

Se utilizó agua de mar procedente de la zona del Callao, Perú, la cual fue filtrada en primera instancia por un filtro rápido de $45 \mu \mathrm{m}$ a $50 \mu \mathrm{m}$. Para luego pasar por tres microfiltros de fibra de algodón con poros de 10.5 y $1 \mu \mathrm{m}$, respectivamente. Finalmente pasó por un sistema de esterilización UV. El sedimento marino empleado fue procedente del lugar de la colecta. Este sustrato fue lavado con agua de mar UV filtrada, luego tamizado con una malla de 1000 um y finalmente esterilizado en un horno a $120^{\circ} \mathrm{C}$ por $24 \mathrm{~h}$.

\section{Preparación de concentraciones de cromo}

La solución madre del cromo se preparó pesando $3.05 \mathrm{~g}$ de $\mathrm{K}_{2} \mathrm{Cr}_{2} \mathrm{O}_{7}$, la cual fue secada en el horno a 20 ${ }^{\circ} \mathrm{C}$ por $1 \mathrm{~h}$, y luego fue colocada en una cámara desecadora por $30 \mathrm{~min}$. Finalmente se pesó $2.11 \mathrm{~g}$ de la sal y se disolvió en $1 \mathrm{~L}$ de agua de mar UV filtrada a $0.45 \mu \mathrm{m}$ en una fiola, esto equivale a una concentración nominal de $193.3 \mathrm{mg}$ de $\mathrm{Cr}^{+6} \mathrm{~kg}^{-1}$ de sedimento. Se prepararon cinco diluciones a partir de la solución stock y distribuidas en volúmenes de 100 $\mathrm{mL}$ para cada acuario, luego se procedió agregar 386.6 $\mathrm{g}$ de sedimento seco dejando homogeneizar y estabilizar la arena húmeda por $1 \mathrm{~h}$, después se añadió $1.1 \mathrm{~L}$ de agua de mar UV para conservar una proporción de 1:3 p/p de sedimento:agua, con la ayuda de una manguera y un disco de polietileno para evitar la perturbación del sedimento (APHA, 1995). Finalmente se dejó con aireación por $1 \mathrm{~h}$ para estabilizar y equilibrar el medio. Las concentraciones nominales finales del cromo empleadas se encontraron en el rango de 46.41 a $193.3 \mathrm{mg}$ de $\mathrm{Cr}^{+6} \mathrm{~kg}^{-1}$ de sedimento seco. El factor de dilución empleado fue de 0.7 .

\section{Pruebas ecotoxicológicas}

Los bioensayos se realizaron con adultos de $D$. obesulus de $2.3 \pm 0.4 \mathrm{~g}(1.51-2.93 \mathrm{~g} ; \mathrm{n}=18)$ de peso húmedo y $22.4 \mathrm{~mm} \pm 1.5 \mathrm{~mm}(19.1 \mathrm{a} 24.5 \mathrm{~mm} ; \mathrm{n}=$ 18). El peso seco fue de $0.14 \pm 0.01 \mathrm{~g}(0.10-0.16 \mathrm{~g}$; $\mathrm{n}=20$ ). Se usó 40 individuos al azar por concentración y 10 por cada una de las cuatro réplicas, para luego contar el número de sobrevivientes al cabo de 24,48 y $96 \mathrm{~h}$. Los organismos no se alimentaron durante el bioensayo. Al realizar la prueba de toxicidad, los individuos se consideraron muertos cuando tenían las valvas completamente cerradas y no eran capaces de contraer el talo y el sifón al ser pinchadas con un estilete. La mortalidad se confirmó con la ayuda de un estereoscopio binocular al no visualizar movimiento al ser colocadas en agua de mar UV aireada por espacio de $1 \mathrm{~h}$. Con el fin de evaluar efectos en el comportamiento de enterramiento en el sustrato arenoso, se registró si éstos estaban sobre el sustrato (superficiales, no enterrados en el sustrato), semienterrados (hasta un $90 \%$ de organismo enterrado en el sustrato) y enterrados (totalmente enterrados en el sustrato). Las condiciones y criterios de aceptabilidad del bioensayo con $D$. obesulus son indicados en la Tabla 1.

\section{Análisis de datos}

La prueba de toxicidad estática-aguda a las $24 \mathrm{~h}$, $48 \mathrm{~h}$ y $96 \mathrm{~h}$ para $D$. obesulus se realizó en cuatro repeticiones con cinco concentraciones nominales, más el control en un diseño en bloque completamente randomizado (DBCR) (Iannacone et al., 2000). La eficacia de los tratamientos se evalúo a través de un análisis de varianza (ANDEVA) en caso de existir diferencias significativas entre las repeticiones se procedió a realizar la prueba de Tukey. Para evaluar efectos subletales en el comportamiento de enterramiento se empleó un ANDEVA para determinar si existían diferencias entre las concentraciones empleadas a $24 \mathrm{~h}$ y $48 \mathrm{~h}$ de exposición. La estimación de la concentración letal media $\left(\mathrm{CL}_{50}\right)$ y sus respectivos límites confianza al 95\% se calcularon usando el programa estadístico computarizado PROBIT, versión 1.5 de la USEPA (Weber, 1993).

\section{Resultados}

\section{Tasa de filtración}

La evaluación de tres microalgas $S$. costatum, $D$. tertiolecta y C. gracilis para determinar la mayor tasa de filtración en $D$. obesulus, mostró a los $30 \mathrm{~min}$ de evaluación la siguiente secuencia de mayor a menor 
tasa de filtración: S. costatum $(46.9 \%)>$ Ch. gracilis $(28.9 \%)>D$. tertiolecta $(25.3 \%)$. En cambio, opuestamente a 60 min de exposición el patrón fue: $D$. tertiolecta $(53.2 \%)>$ Ch. gracilis $(48.4 \%)>S$. costatum (36.7\%). Por ende, para las condiciones de aclimatación y cría de $D$. obesulus se empleó a la microalga marina $D$. tertiolecta.

Tabla 1. Condiciones y criterios de aceptabilidad recomendadas para conducir la prueba de toxicidad con D. obesulus.

\begin{tabular}{|c|c|}
\hline Parámetros & Condiciones \\
\hline Tipo de bioensayo & Estático - agudo \\
\hline Duración de la prueba & $96 \mathrm{~h}$ \\
\hline Serie de diluciones & $\begin{array}{l}\text { Un control y por lo menos cinco } \\
\text { concentraciones (factor de } \\
\text { dilución } 0.7 \text { ) }\end{array}$ \\
\hline Tóxico & $\mathrm{K}_{2} \mathrm{Cr}_{2} \mathrm{O}_{7}\left(\mathrm{Cr}^{+6}\right)$ \\
\hline Temperatura $\left({ }^{\circ} \mathrm{C}\right)$ & 17 a 18 \\
\hline Salinidad & 34.3 a 34.9 \\
\hline $\mathrm{pH}$ & $\approx 8$ \\
\hline Calidad de luz & $\begin{array}{l}\text { Iluminación ambiental del } \\
\text { laboratorio }\end{array}$ \\
\hline Fotoperíodo & $10 \mathrm{~h}$ de luz / $14 \mathrm{~h}$ de oscuridad \\
\hline Tamaño de envase (L) & 1.5 \\
\hline $\begin{array}{l}\text { Cantidad de sedimento ( } \mathrm{g} \text { peso } \\
\text { seco) }\end{array}$ & 385 a 390 \\
\hline Volumen de agua (L) & 1.2 \\
\hline $\begin{array}{l}\text { Longitud de los organismos } \\
\qquad(\mathrm{mm})\end{array}$ & $\begin{array}{l}\text { Adultos de tamaño promedio } 21.1 \\
\qquad \mathrm{~mm}\end{array}$ \\
\hline $\begin{array}{l}\mathrm{N}^{\circ} \text { de organismos por } \\
\text { concentración }\end{array}$ & 10 \\
\hline $\begin{array}{l}\mathrm{N}^{\circ} \text { de replicas por } \\
\text { concentración }\end{array}$ & 4 \\
\hline Oxígeno $\left(\mathrm{mg} \cdot \mathrm{L}^{-1}\right)$ & $\approx 7$ \\
\hline $\begin{array}{c}\text { Tiempo de observación en } \\
\text { acuario }\end{array}$ & $\begin{array}{c}1 \mathrm{~h} \text { de observación al } \\
\text { estereoscopio }\end{array}$ \\
\hline Respuesta letal & $\begin{array}{c}\text { Mortalidad (ausencia de } \\
\text { movimiento del talo y valvas) }\end{array}$ \\
\hline Criterio de aceptabilidad & $\begin{array}{c}\text { Sobrevivencia del } 90 \% \text { en los } \\
\text { controles }\end{array}$ \\
\hline
\end{tabular}

\section{Pruebas ecotoxicológicas}

Las $\mathrm{CL}_{50}$ de cromo y sus limites de confianza inferior y superior usando $D$. obesulus en sedimento marino fueron: $410.4 \mathrm{mg} \cdot \mathrm{kg}^{-1}\left(376.3 \mathrm{mg} \cdot \mathrm{kg}^{-1}\right.$ y 538.8 $\left.\mathrm{mg} \cdot \mathrm{kg}^{-1}\right) ; 156.1 \mathrm{mg} \cdot \mathrm{kg}^{-1}\left(146.9 \mathrm{mg} \cdot \mathrm{kg}^{-1}\right.$ y $167.3 \mathrm{mg} \cdot \mathrm{kg}^{-}$ $\left.{ }^{1}\right)$, y $61.12 \mathrm{mg} \cdot \mathrm{kg}^{-1}\left(56.36 \mathrm{mg} \cdot \mathrm{kg}^{-1}\right.$ y $\left.65.50 \mathrm{mg} \cdot \mathrm{kg}^{-1}\right)$ a $24 \mathrm{~h}, 48 \mathrm{~h}$ y $96 \mathrm{~h}$ de exposición, respectivamente. La Figura 1 muestra la relación entre la concentración del cromo $\left(\mathrm{mg} \cdot \mathrm{kg}^{-1}\right)$ y la mortalidad de D. obesulus (\%) a 24 h, 48 h y 96 h de exposición.

\section{Comportamiento de enterramiento}

La Tabla 2 muestra de manera comparativa el comportamiento de D. obesulus en las cinco concentraciones a 24 y 48 h de exposición. La capacidad de semienterramiento y enterramiento en el control suman el $95 \%$ y $90 \%$ a las 24 y $48 \mathrm{~h}$ de exposición, respectivamente. Para la menor concentración $46.41 \mathrm{mg} \cdot \mathrm{kg}^{-1}$, la suma de la capacidad de semienterramiento y enterramiento fue de $77.5 \%$ y $67.5 \%$ a 24 y 48 h, respectivamente. Para la mayor concentración $193.30 \mathrm{mg} \cdot \mathrm{kg}^{-1}$, la suma de la capacidad de semienterrase y enterramiento es de $50 \%$ y $17.50 \%$ a las 24 y $48 \mathrm{~h}$ de exposición, respectivamente. A las $24 \mathrm{~h}$ y $48 \mathrm{~h}$ de exposición se encontró diferencias significativas entre el control con la concentración de $46.41 \mathrm{mg} \cdot \mathrm{kg}^{-1}$, en el comportamiento de no enterramiento (superficial), semienterramiento, y enterramiento con la concentración de $46.41 \mathrm{mg} \cdot \mathrm{kg}^{-1}$. Esto nos indica una inhibición en la capacidad de enterramiento de $D$. obesulus a medida que aumenta la concentración y el tiempo de exposición (Tabla 2).

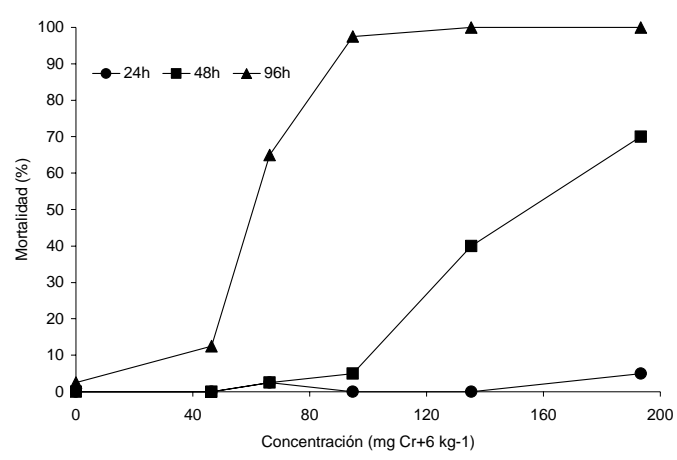

Figura 1. Efecto del cromo en sedimento en la mortalidad de Donax obesulus a 24 h, 48 h y 96 h de exposición.

\section{Discusión}

Los organismos bentónicos están en contacto íntimo con el sedimento, ya sea en la superficie o como excavadores, y algunos incluso se alimentan de él, por ello son los animales muy usados en las pruebas de toxicidad para sedimentos (Iannacone et al., 1998). Se utilizan un gran número de especies de diferentes grupos taxonómico como amphipoda, annelida, insecta, mollusca y nemátoda (Del Valls \& Corandi, 2000). En nuestro caso empleamos al bivalvo D. obesulus (Iannacone \& Alvariño, 1998, 1999). Del Valls \& Corandi (2000), señala el uso de la almeja Ruditapes philippinarum (Adams \& Reeve 1850) en una prueba de toxicidad de sedimento. Nosotros sugerimos a la "concha mariposa" Donax obesulus, por ser una especie propia del Perú.

Existen criterios de letalidad, para reconocer a los organismos bivalvos muertos, en especies como Argopecten purpuratus (Lamark 1819) cuando tengan las valvas abiertas y no las cierren al ser tocadas con un pincel (Sánchez \& Vera, 2001). En nuestro caso el efecto letal para $D$. obesulus se determinó cuando tuvieron las valvas cerradas y no eran capaces de contraer el talo y el sifón al ser pinchadas con estilete. Los efectos subletales, como la capacidad de enterramiento a 24 y 48 horas de exposición se ven 
inhibidos, debido al aumento de la concentración del tóxico y al tiempo de exposición (Del Valls \& Corandi, 2000). Los criterios para evaluar el procedimiento de bioensayo de toxicidad, no solo involucra la sensibilidad, si no también, la facilidad de uso, costo y realismo ecológico (Crisinel et al., 1994). Nuestro estudio con el pelecípodo D. obesulus muestra el fácil manejo en el laboratorio, a menor tamaño de los especímenes, mejor el método (Iannacone \& Alvariño, 1998). El costo que incluye principalmente el acondicionamiento y aclimatación de los organismos es económico. El realismo ecológico favorece al molusco ya que cumple un rol importante en el ecosistema arenoso marino.

herramientas para evaluar el riesgo de los sedimentos. $\mathrm{La}$ evaluación de respuestas subletales provee información valiosa para evaluar la toxicidad de los sedimentos (Norberg-King et al., 2006). En el presente estudio la evaluación del comportamiento de enterramiento de $D$. obesulus por efecto del cromo proporciona mayor sensibilidad que el criterio de mortalidad, al seguir una secuencia de condiciones de enterramiento, luego semienterramiento y finalmente no enterramiento (superficial) de $D$. obesulus con el aumento de la concentración del cromo en el sedimento (Tabla 2).

Arrascue et al. (2001), indican que en el sistema legal internacional, las pruebas de toxicidad para evaluar sedimentos con invertebrados acuáticos son

Tabla 2. Comportamiento de enterramiento de D. obesulus frente al cromo.

\begin{tabular}{|c|c|c|c|c|c|c|c|c|}
\hline \multirow{3}{*}{$\begin{array}{c}\mathrm{mg} \mathrm{Cr}^{+6} \\
\mathrm{~kg}^{-1} \text { de } \\
\text { sedimento }\end{array}$} & \multicolumn{8}{|c|}{ Comportamiento $(\%)$} \\
\hline & \multicolumn{4}{|c|}{$24 \mathrm{~h}$} & \multicolumn{4}{|c|}{$48 \mathrm{~h}$} \\
\hline & Superficie & $\begin{array}{c}\text { Semi - } \\
\text { Enterrado }\end{array}$ & Enterrado & Muerto & Superficie & $\begin{array}{c}\text { Semi - } \\
\text { Enterrado }\end{array}$ & Enterrado & Muerto \\
\hline 0 & $5.0 \mathrm{a}$ & $40.0 \mathrm{a}$ & $55.0 \mathrm{a}$ & $0.0 \mathrm{a}$ & $10.0 \mathrm{a}$ & $45.0 \mathrm{c}$ & $45.0 \mathrm{~b}$ & $0.0 \mathrm{a}$ \\
\hline 46.41 & $22.5 \mathrm{a}$ & $72.5 b$ & $5.0 \mathrm{~b}$ & $0.0 \mathrm{a}$ & $32.5 b$ & $62.5 \mathrm{~d}$ & $5.0 \mathrm{a}$ & $0.0 \mathrm{a}$ \\
\hline 66.30 & $25.0 \mathrm{~b}$ & $72.5 b$ & $0.0 \mathrm{~b}$ & $2.5 \mathrm{a}$ & $65.0 \mathrm{c}$ & $32.5 b c$ & $0.0 \mathrm{a}$ & $2.5 \mathrm{a}$ \\
\hline 94.71 & $42.5 \mathrm{bc}$ & $57.5 \mathrm{ab}$ & $0.0 \mathrm{~b}$ & $0.0 \mathrm{a}$ & $75.0 \mathrm{c}$ & $20.0 \mathrm{ab}$ & $0.0 \mathrm{a}$ & $5.0 \mathrm{a}$ \\
\hline 135.31 & $50.0 \mathrm{c}$ & $50.0 \mathrm{ab}$ & $0.0 \mathrm{~b}$ & $0.0 \mathrm{a}$ & $50.0 \mathrm{c}$ & $10.0 \mathrm{a}$ & $0.0 \mathrm{a}$ & $40.0 \mathrm{~b}$ \\
\hline 193.30 & $45.0 \mathrm{bc}$ & $50.0 \mathrm{ab}$ & $0.0 \mathrm{~b}$ & $5.0 \mathrm{a}$ & $12.5 \mathrm{ab}$ & $17.5 \mathrm{a}$ & $0.0 \mathrm{a}$ & $70.0 \mathrm{c}$ \\
\hline
\end{tabular}

Letras minúsculas iguales en una misma columna indican que los promedios son estadísticamente semejantes a un $\mathrm{P}<0.05$

El cromo es encontrado en el ambiente, en dos diferentes formas, siendo la forma hexavalente la más soluble, tóxica y de mayor interés en el ambiente. El $\mathrm{Cr}^{+6}$ causa estrés oxidativo, daño al ADN y perturbación de la expresión genética de las especies asociadas acuáticas (Roling et al., 2006). En el Perú, no existen estándares de calidad ambiental para metales en sedimentos marinos. El valor guía propuesto por Long et al. (1995) para el cromo en sedimentos acuáticos es de $370 \mathrm{mg} \cdot \mathrm{kg}^{-1}$. Los resultados obtenidos en términos de $\mathrm{CL}_{50} 48 \mathrm{~h}$ y $96 \mathrm{~h}$ para D. obesulus, expuestos al cromo, fueron $57.8 \% \mathrm{y}$ $83.5 \%$ menores que el valor guía, respectivamente. Sin embargo, Becker et al. (2006) recientemente han propuesto un valor de no efecto del cromo en sedimentos de $1310 \mathrm{mg} \cdot \mathrm{kg}^{-1}$ evaluando el efecto del cromo en la mortalidad de los anfípodos Ampelisca abdita Mills 1964 y Leptocheirus plumulosus Shoemaker 1932. Debido al poco tiempo de estabilización del cromo en el sedimento ( $1 \mathrm{~h})$, es posible que existan diferencias entre las concentraciones nominales y las reales.

El número de métodos para evaluar la toxicidad de sedimentos se ha incrementado en la última década, lo cual refleja la necesidad de desarrollar mejores las que presentan mayor validez. En el presente estudio sugerimos emplear el invertebrado $D$. obesulus para evaluación del sedimento directo, ya que los sedimentos pueden presentar concentraciones de metales pesados tres órdenes de magnitud mayor que en el agua (Gaete et al., 1996).

De esta forma los procedimientos estandarizados aprobados por la USEPA (Agencia de Protección del Medio Ambiente de los EEUU), adaptados a las especies de bivalvos locales, sugieren emplear equivalentes ecológicos de cada latitud (Cripe, 2006). En nuestro caso D. obesulus es una especie de amplia distribución y adaptada al mesolitoral e infralitoral arenoso peruano, por lo cual es necesario continuar con los programas de evaluación de efectos de la contaminación marina sobre los organismos, como un elemento indicativo para determinar las normas de calidad ambiental, así como el de establecer a través de las pruebas de toxicidad estándares de calidad ambiental (USEPA, 1993).

\section{Conclusiones}

La concentración letal media $\left(\mathrm{CL}_{50}\right)$ del metal de referencia cromo $\mathrm{Cr}^{+6}$ en el sedimento marino usando a D. obesulus fue de $61.12 \mathrm{mg} \cdot \mathrm{kg}^{-1}$ a $96 \mathrm{~h}$ de exposición. El cromo presenta un notable efecto subletal sobre la capacidad de enterramiento de $D$. obesulus a las $48 \mathrm{~h}$ de exposición. El protocolo usando D. obesulus tiene como ventaja, además de su sensibilidad, el fácil uso y la simplicidad en la lectura, su costo y su realismo ecológico. 


\section{Agradecimientos}

Al Sr. Edgardo Alarcón por las sugerencias al resumen del trabajo de investigación presentado en el II congreso de la Red Mundial de Científicos Peruanos. Al Dr. Jorge Tam del Centro de Modelado del IMARPE por las sugerencias para mejorar el proyecto de investigación, al Tco. Edwin Pinto y al Tco. José Ortega, personal del Laboratorio de Ecotoxicología Acuática del IMARPE por la ayuda en el desarrollo de este trabajo de investigación. Al Sr. Valentín Mogollón docente de la facultad de pesquería y oceanografía (UNFV) y al Blgo. Leonardo Romero docente de la facultad de ciencias biológicas (UNMSM), por la bibliografía proporcionada y la identificación taxonómica de los organismos prueba. A Tatiana Cáceres y a Celer Pantoja, por el apoyo para la culminación de la presente investigación.

\section{Literatura citada}

Álamo V. \& Valdivieso V. 1997. Lista sistemática de moluscos del Perú. $2^{\text {do }}$ Ed. IMARPE. Callao-Perú. Alayo M. \& Iannacone J. 2000. La microalga marina Skeletonema costatum (Greville) Cleve como bioensayo alternativo para la evaluación del cromo. Winay Yachay. 4: 69-75.

Alayo M. \& Iannacone J. 2000. La microalga marina Skeletonema costatum (Greville) Cleve como bioensayo alternativo para la evaluación del cromo. Winay Yachay. 4: 69-75.

Alayo M., Iannacone J. \& Arrascue A. 2004. Sensibilidad al cromo: microbiopruebas con las diatomeas marinas Isochrysis galbana Parke y Chaetoceros gracilis Schütt. Ecología Aplicada. 3: 154-161.

APHA (American Public Health Association), AWWA (American Water Works Association), WPCF (Water Pollution Control Federation). 1995. Standard methods for examination of water and wastewater. $19^{\text {th }}$ Ed. American Health Association. Washington D.C.

Arrascue A., Iannacone J., Alvariño L., Basilio S. \& Lazcano C. 2001. El insecto Chironomus calligraphus Goeldi y la bacteria Escherichia coli como ensayos ecotoxicológicos para evaluar sedimentos elutriados dulceacuícolas. Revista Peruana de Entomología. 42: 169-173.

Becker D.S., Long E.R., Proctor D.M. \& Ginn T.C. 2006. Evaluation of potential toxicity and bioavailability of chromium in sediments associated with chromite ore processing residue. Environmental Toxicology and Chemistry. 25: 2576-2583.

Chai Y., Kochetkov A. \& Reible D.D. 2006. Modeling biphasic sorption and desorption of hydrophobic organic contaminants in sediments. Environmental Toxicology and Chemistry. 25: 3133-3140.

Coan E.V. 1983. The Eastern Pacific Donacidae. Veliger. 25: 273-298.
Cripe G.M. 2006. Contaminated sediment testing with the bivalve Mulinia lateralis. Culture refinement for organism availability. Environmental Toxicology and Chemistry. 25: 1332-1336.

Crisinel A., Delaunay L., Rossel D., Tarradellas J., Meyer H., Sarah H., Vogel P., Delisle C. \& Bloise C. 1994. Cyst-based ecotoxicological tests using anostracans: comparison of two species of Streptocephalus. Environmental Toxicology and Water Quality. 9: 317-324.

Del Valls A., Forja J. \& Gómez A. 1998. El uso de análisis multivariante en la unión de datos de toxicidad y contaminación para establecer guías de calidad de sedimento: un ejemplo en la bahía de Cádiz (España). Ciencias del Mar. 24: 127-154.

Del Valls T. \& Corandi, M. 2000. Avances en ecotoxicología marina: comparación entre test de laboratorio y estudios in situ para la evaluación de la calidad ambiental de los sedimentos. Ciencias Marinas. 26:39-64.

Dornfeld E., Espíndola E.L.G., Fracácio R., Rodríguez B.K. \& Novelli A. 2006. Comparação do bioensaios laboratoriais e "in situ" utilizando Chironomus xanthus na avaliacao da toxicidade de sedimentos do rio Monjolinho (Sao Carlos, SP). Journal of the Brazilian Society of Ecotoxicology. 1: 161-166.

Estrella C., Guevara-Carrasco R., Ávila W., Palacios J. \& Medina A. 2000. Informe estadístico anual de los recursos hidrobiológicos de la pesca artesanal por especies, artes, caletas y meses durante el segundo semestre de 1999. Informe Instituto del Mar del Perú. 151: 1-194.

Gaete H., Silva J., Riveros A., Soto E., Troncoso L., Bay-Sehmidt E. \& Larrain A. 1996. Efecto combinado y riesgo ecológico de las concentraciones de $\mathrm{Zn}, \mathrm{Cu}$ y $\mathrm{Cr}$ presente en el Puerto de San Vicente, Chile. Gayana Oceanológica. 4: 99-107.

Huaraz F. \& Yshiyama V. 1980. Madurez sexual de la "Concha Mariposa" (Donax peruvianus) de la playa de Jahuay, Ica, Perú. Revista Ciencias UNMSM. 72: 47-56.

Iannacone J. \& Alvariño L. 1998. Ecotoxicidad aguda del Zinc sobre el "Guppy" Poecilia reticulata Wiñay yachay. 2(3): 67-74.

Iannacone J. \& Alvariño L. 1999. Ecotoxicidad aguda de metales pesados empleando juveniles el caracol de agua dulce Physa venustula (Gould, 1847) (Mollusca). Gayana. 63: 101-110.

Iannacone J. \& Alvariño L. 2003. Efecto ecotoxicológico agudo del mercurio sobre larvas del "muy muy" Emerita analoga (Stimpson) (Decapada: Hippidae) procedentes de cuatro localidades de Lima. Ecología Aplicada. 2: 111 115 . 
Iannacone J., Alvariño L., Caballero C. \& Sánchez J. 2000. Cuatro ensayos ecotoxicológicos para evaluar lindano y clorpirifos. Gayana. 64:139-146.

Iannacone J., Alvariño L. \& Dale W. 1998. Pruebas ecotoxicológicas como herramienta para la evaluación del impacto ambiental en los ecosistemas acuáticos. Boletín de Lima (Perú). 13: 53- 68.

Long E.R., MacDonald D.D., Smith S.L., Calder F.D. 1995. Incidence of adverse biological effects within ranges of chemical concentrations in marine and estuarine sediments. Environmental Management. 19: 81-97.

Malnati F.L. 1982. La Contaminación de Aguas en el Perú. Boletín de Lima (Perú). 21: 78-89.

Norberg-King T.J., Sibley P.K., Burton G.A., Ingersoll C.G., Kemble N.E., Ireland S., Mount D.R. \& Rowland C.D. 2006. Interlaboratory evaluation of Hyalella azteca and Chironomus tentans short-term and long-term sediment toxicity tests. Environmental Toxicology and Chemistry. 25: 1662-1674.

Ong E. \& Din Z. 2001. Cadmium, copper and zinc toxicity to the clam, Donax faba C. and the cockle, Anadara granosa L. Bulletin of Environmental Contamination and Toxicology. 66: 86-93.

Paredes C., Huaman P., Cardozo F., Vivar R. \& Vera V. 1999. Estado actual del conocimiento de los moluscos acuáticos del Perú. Revista Peruana de Biología. 6: 5-47.

Paredes C. \& Cardozo F. 2001. El género Donax en la costa peruana (Bivalvia: Tellinoidea). Revista Peruana de Biología. 8: 83-93.

Portela C.M.S., Arenzon A. \& Raya-Rodriguez M.T. 2006. Avaliação ecotoxicológica do sedimento do arroio Sapucaia, RS, Brasil, através de ensaios de toxicidade utilizando diferentes amostradores e organismos-teste. Journal of the Brazilian Society of Ecotoxicology. 1: 119-122.
Roling J.A., Bain L.J., Gardea-Torresdey J., Bader, J. \& Baldwin W.S. 2006. Hexavalent chromium reduces larvae growth and alters gene expression in mummichog (Fundulus heteroclitus). Environmental Toxicology and Chemistry. 25: 2725-2733.

Sánchez R.G. \& Vera D.G. 2001. Manual Introductorio de ecotoxicología acuática. Informe Instituto del Mar del Perú. 161: 1-40.

Talledo R. 1980. Algunas consideraciones bioecologicas de Donax peruvianus, Deshayes 1855. Tesis para optar el titulo de Licenciado en Biología - Pesquera. Universidad Nacional Pedro Ruiz Gallo. Lambayeque. Perú.

Talledo R., Yshiyama V. \& Tarazona J. 1987. Reproducción de Donax peruvianus, Deshayes 1855 en el área de la caleta San José y playa Naylamp, Lambayeque. Biota. 93: 4-16.

Tarazona J., Paredes C. \& Igreda M. 1986. Estructura de macrobentos en las playas arenosas en la zona de Lima. Perú. Revista de Ciencias UNMSM. 74: 103-116.

USEPA, 1993. Guidelines for deriving site-speficic sediment quality criteria for the protection of benthic organisms. EPA-822-R-03-017, U.S. Environmental Protection Agency, Office of Science and Technology, Health and Ecological Criteria Division, Washington, D.C.

Vera G., Tam J. \& Pinto E. 2001. Pruebas ecotoxicológicas con cadmio y cromo usando post larvas de pejerrey Odontesthes (Austromenidia) regia regia Humboldt. Revista Peruana de Biologia. 8: 125-135.

Weber C. 1993. Methods for measuring the acute toxicity of effluents and receiving waters to freshwater and marine organisms. EPA/600/490/027F.

\footnotetext{
${ }^{1}$ Laboratorio de Ecofisiología Animal. Facultad de Ciencias Naturales y Matemática. Universidad Nacional Federico Villarreal. Calle San Marcos 383, Pueblo Libre. Correo electrónico: juliolacmas@ hotmail.com

${ }^{2}$ Laboratorio de Invertebrados. Facultad de Ciencias Biológicas. Universidad Ricardo Palma. Av. Benavides 5440, Lima 33, Perú. Correo electrónico: joseiannacone@yahoo.es

${ }^{3}$ Línea de investigación en Ecotoxicología. Instituto del Mar del Perú. Correo electrónico: gioverad@yahoo.com
} 